\title{
THE ECOLOGY OF MICROSPORUM CANIS BODIN IN NEW ZEALAND
}

\author{
BY MARY J. MARPLES \\ Department of Microbiology, University of Otago, New Zealand
}

The most common cause of scalp and body ringworm in New Zealand is the pathogenic fungus Microsporum canis. This organism is primarily a parasite of domestic pets, and has been shown by many authorities to lose its infectivity after relatively few human passages so that it causes small family outbreaks, rather than widespread epidemics.

The present investigation has been concerned with epidemiological and ecological problems, rather than those of diagnosis and therapy. An attempt has been made to determine the types of transmission of infection which can occur, and to obtain information about the network of infection which must exist among human and animal hosts, parasitized by this fungus. La Touche $(1955 b)$ has stressed the importance of domestic animals as a reservoir of the dermatophytes, and a closer study of the types of contact with animals which resulted in human infections seemed worth attempting.

In a previous investigation (Marples, 1951) it was shown that the course of a scalp infection caused by $M$. canis could follow three different clinical patterns. The lesions could remain localized with no spread of infection, or there could be local spread with the appearance of small satellite lesions, or the infection could become generalized, so that single fluorescent hairs would be found, scattered over large areas of the scalp. This report also embodies the results of an endeavour to relate these clinical patterns to various factors, such as the source of infection, and the age and sex of the patient.

The survey extends over the years 1952-5, during which period the number of cases per year has remained reasonably constant. The patients have all been treated by topical applications only. The clinical patterns and seasonal incidence already reported (Marples, 1951) have been found to occur during the present study.

\section{MATERIAL AND METHODS}

The children included in this investigation were drawn from Dunedin and its suburbs, and were those sent for diagnosis to the bi-weekly mycological diagnostic clinic, held in the Otago Medical School. These children were sent in by the Dunedin Public Hospital Outpatients Departments, the School Medical Officers of the district, by private practitioners and dermatological specialists. They appear to provide a reasonable cross-section of the cases of scalp ringworm occurring in the area.

At the first visit each child was examined under the Wood lamp, and the site and number of fluorescent lesions was recorded. Fluorescent hairs were inoculated on 
to Sabouraud glucose-agar slopes, and incubated at room temperature. Those cases from which a strain of $\boldsymbol{M}$. canis was isolated were included in the survey.

At the same visit the following facts were recorded on a punch card:

Name, sex, age and address of the child.

Occupation of the breadwinner of the family.

History of the onset of the infection and of its duration before attendance at the diagnostic clinic.

The number of adult and juvenile members of the family and the occurrence of tinea capitis or tinea corporis among them.

The possible sources of infection with special reference to animal and human contacts.

Each patient was subsequently examined at fortnightly intervals, under the Wood light and the clinical progress was recorded.

The child was discharged as cured when no fluorescent hairs were seen and when microscopic examination of hairs failed to show fungal spores.

The other children in the family and domestic pets were examined wherever possible. The house was then visited and notes were made on the general condition of the premises. The mother was questioned on her methods of house cleaning and on the toilet hygiene of the children. The home conditions were grouped as good, fair or poor, using the following criteria:

\section{Home conditions good}

Children have separate bedrooms or separate beds in a room shared with only one sibling.

House clean and tidy. Garden well cultivated.

Vacuum cleaner used regularly, weekly or more often.

Children bathed daily or every second day.

Children's hair washed once a week or more often.

Domestic pets receiving affection and care.

\section{Home conditions fair}

More than two children occupying separate beds in the same bedroom.

House and garden reasonably tidy.

Vacuum cleaner not used, or used irregularly.

Children bathed only once a week.

Hair washed irregularly.

Family not particularly interested in domestic pets.

\section{Home conditions poor}

Several children and/or adults accommodated in beds or bunks in the same room. House old, dirty, untidy and dilapidated and sometimes without a bathroom. Garden littered with rubbish and poorly fenced.

Vacuum cleaner not used.

Bathing of children and hair washing irregular.

Animals roaming about the premises and not clearly owned or acknowledged by the family. 
It was unfortunately not possible to obtain the complete information about each family. Not all the houses were visited nor was the status of the breadwinner always recorded. Many of the children ceased to visit the clinic before the infection had completely disappeared, so that the duration of the infection could not be determined. The greatest difficulty, however, lay in the examination of domestic pets. New Zealand parents are aware that cats are a common source of fungous infection, and in many of the families the cat had already been destroyed before the child paid his first visit to the clinic.

\section{RESULTS OF THE INVESTIGATION}

These results are arranged with reference to the parasite, the human and other hosts, the sources of infection and the transmissions that were observed.

\section{(1) The parasite}

Little need be said about the parasite. The colonies on Sabouraud agar were visible 3-4 days after inoculation, and the majority of strains produced yellow pigment in the medium and abundant thick-walled spindle-shaped macroconidia. A few strains did not produce pigment and were identified as $M$. canis var. album, the variant named by Walker (1950). Two dysgonic strains with slow growth and delayed production of macroconidia were isolated. In three subjects, no fluorescence under the Wood lamp was seen throughout the clinical course, and the progress had to be followed by direct and cultural examination.

\section{(2) The human host}

The patients were drawn from 138 families and included 171 patients with tinea capitis. In eight families the scalp was never invaded and only the lesions of tinea corporis occurred. The distribution of infections are shown in Table 1. Of the 171 cases of tinea capitis $111(65 \%)$ were males. The ages ranged from less than 1 year to 12 years, the youngest child included being 9 months of age. The infection was almost equally distributed in the earlier age group, as of the 87 children whose ages ranged from 0 to 4 years, 46 were males and 41 females. The females in the older

Table 1. The age distribution and clinical features of 171 cases of tinea capitis caused by Microsporum canis

\begin{tabular}{|c|c|c|c|c|c|c|}
\hline & M. & $\%$ & F. & $\%$ & Total & $\%$ \\
\hline No. of cases & 111 & $64 \cdot 9$ & 60 & $35 \cdot 1$ & $17 \mathrm{I}$ & - \\
\hline Age group: $0-4$ years & 46 & $41 \cdot 4$ & 41 & $68 \cdot 3$ & 87 & $50 \cdot 9$ \\
\hline $5-9$ years & 54 & $48 \cdot 7$ & 19 & $31 \cdot 7$ & 73 & $42 \cdot 7$ \\
\hline$>10$ years & 11 & $9 \cdot 9$ & 0 & 0 & 11 & $6 \cdot 4$ \\
\hline Tinea corporis also present & 34 & $30 \cdot 6$ & 26 & $43 \cdot 3$ & 60 & $35 \cdot 1$ \\
\hline No. of clinical patterns known & 104 & -_. & 51 & - & 155 & - \\
\hline Local lesions only & 25 & 24 & 14 & $27 \cdot 4$ & 39 & $25 \cdot 2$ \\
\hline Local spread of infection & 52 & 50 & 27 & $52 \cdot 9$ & 79 & 51 \\
\hline Generalized spread of infection & 27 & 26 & 10 & $19 \cdot 7$ & 37 & $23 \cdot 8$ \\
\hline Severe inflammatory reaction & 28 & $26 \cdot 9$ & 7 & $13 \cdot 7$ & 35 & $22 \cdot 6$ \\
\hline
\end{tabular}


age groups were less frequent. Of the 73 patients whose age group was 5-9 years, only 19 were females. No cases of scalp ringworm in girls over the age of 10 years were recorded, but 11 boys of more than 10 years had scalp infections. It would appear that girls become progressively less susceptible to scalp infections with age. It seems unlikely that this is a true difference in susceptibility, and it is more probable that the longer hair of girls makes it difficult for the parasite to establish itself in the surface layers of the scalp. The proportion of male and female infections with M. canis is not the same as that due to M. audouini. Schwartz, Peck, Botvinick, Liebowitz \& Frasier (1946) report that more than $90 \%$ of their 565 cases of scalp ringworm occurred in males.

\section{CLINICAL PATTERNS}

The clinical course of the infection could be followed in 155 subjects, although not all of these children attended the clinic until they could be discharged as cured. Of these children, 39 had lesions which showed no spread, 79 had local spread with the development of satellite patches around the original lesions and 37 showed a generalized spread, involving almost the whole of the scalp. The distribution of these clinical patterns is also shown in Table 1.

There was no correlation of the clinical pattern with the sex or age of the patient, nor with the source of infection which will be discussed later. Different forms of topical application did not appear to alter the course of the infection.

\section{DURATION OF INFECTION}

In only 146 children could the infection be followed until complete cure had been effected. Several of the children ceased to attend the clinic at a stage when one or two fluorescent hairs were still present, and some did not pay their first visit until the infection had been established for some time so that the history of onset was too vague to be reliable. Of the infections followed, 34 had a duration of more than 16 weeks, the longest duration being 8 months. In 66 cases the duration was less than 12 weeks, the shortest being 5 weeks. The remaining infections lasted 12-15 weeks. The infection was shortest in the older age groups whose infection remained localized. There was no statistical correlation between source of infection and duration of infection.

\section{INFLAMMATORY REACTIONS}

Most of the lesions showed some mild reddening, but a severe inflammatory reaction early in the infection only occurred in two cases. Severe reactions developed during the course of treatment in $28(27 \%)$ boys and $7(14 \%)$ girls of the groups whose clinical pattern could be followed. The incidence of severe reactions has been more common during 1954 and 1955, and the duration of infections has been shorter during these years. This is probably due to the very general use in this area of the proprietary preparation Sanicyl as a topical application. This preparation appears to excite an inflammatory response fairly rapidly which results in a more rapid cure. 


\section{ASSOCIATED LESIONS OF TINEA CORPORIS}

In $60(35 \cdot 1 \%)$ of the children suffering from tinea capitis, lesions of tinea corporis were also present. These lesions were often seen by the mother before the scalp lesions were noticed. M. canis characteristically causes lesions both in the hair and on the body, and differs in this way from M.audouini, which Walker (1950) and other authorities state rarely invades the glabrous skin.

\section{SOURCES OF INFECTION}

The sources of infection were divided into the following groups:

(1) Definite cat source. A cat was regarded as the source of infection on the following criteria:

Demonstration of the infection by Wood light and/or direct examination of cat hairs at the diagnostic clinic.

Or diagnosis and treatment of the cat by a veterinary surgeon, or a history of the presence of bald patches on a cat obtained shortly before the simultaneous onset of several human infections.

(2) Definite dog source. The same criteria as for the cat were applied to this group.

(3) Definite human source. Cases included in this group were those in which the family pet was checked and found to be free from ringworm and there was a history of very close contact with another known human infection, such as wearing the cap of an infected child.

(4) Probable animal source. This group included cases where the onset of infection in several members of the family was preceeded by contact with a stray kitten, but where there was no confirmation of the infection in the cat.

(5) Probable human source. Children in this group had no obvious animal contact, but had a not very close contact with a human case. Infections in the clipper area which developed within $\mathbf{1 4}$ days from a visit to the barber were also included.

(6) No obvious source of infection. In this group were placed the children whose pets were negative when examined, and whose history gave no evidence of human contact, and whose hair was cut at home.

Based on the above criteria, 42 of the 138 families included in the survey had a definite cat source of infection, 5 had a definite dog source, and an animal was the probable source in a further 28 families. The infection was ascribed to a definite human source in 19 families and probably to human contacts in a further 11, while in 33 families there was no clue as to the source of the infection.

It is obvious that some of the cases included have been ascribed to the wrong source. Many of the children were very young and could not be satisfactorily questioned about playing with stray animals, nor were their mothers necessarily aware of such contacts. Further, although many domestic pets were examined with negative results, infections might have been missed owing to the difficulties of examining thoroughly a large, angry and frightened cat under the Wood lamp. However, such results as have been obtained suggest that the majority of infections in this area are caught from animals. 
During the investigation into the sources of infection of the children studied, evidence for some unusual types of transmission was obtained. In addition to the more obvious spread of infection from animal to human hosts, clinical histories with reliable dates of onset and laboratory findings in animals demonstrated the transfer of the parasite among domestic pets and from children to animals. Evidence for the following kinds of transmission was obtained: cat to child; child to child; child to adult; child to cat; dog to cat; and cat to dog. These findings emphasize the importance of the animal reservoir in $M$. canis infections, and demonstrate the complex ecological relationships of this parasite.

\section{THE INFECTION IN THE ANIMAL HOST}

Although the cat has been regarded as the source of infection of 42 families, only 15 positive infections were seen at the diagnostic clinic. A number of cats suffering from ringworm, but not connected with this survey, have also been examined from time to time. The extent of infection has varied considerably among the cats studied. One very young animal had large areas of the body which were almost

Table 2. The social conditions and sources of infection in 138 families infected with Microsporum canis

\begin{tabular}{lrc} 
& No. & $\%$ \\
No. of families & 138 & - \\
No. of cases of scalp ringworm & 171 & - \\
Siblings in family & 114 & $\mathbf{8 2 \cdot 6}$ \\
Siblings of patient infected & 71 & $62 \cdot 3$ \\
Parents infected & 14 & $10 \cdot 1$ \\
Total social classes known & 95 & - \\
Social class: 0 & 4 & $4 \cdot 2$ \\
\multicolumn{1}{c}{ II } & 12 & $12 \cdot 6$ \\
$\quad$ III & 57 & 60 \\
No. of homes visited & 22 & $22 \cdot 1$ \\
Home conditions: Good & 76 & - \\
$\quad$ Fair & 45 & 58 \\
Definite cat source of infection & 25 & 33 \\
Definite dog source & 6 & 9 \\
Definite human source & 52 & $30 \cdot 5$ \\
Probable animal source & 19 & $3 \cdot 6$ \\
Probable human source & 28 & $\mathbf{1 3 \cdot 9}$ \\
No obvious source of infection & 11 & $\mathbf{2 0} \cdot 3$ \\
& 33 & $23 \cdot 9$
\end{tabular}

naked and fluorescent hairs or stumps covered most of the surface, so that under the Wood lamp it appeared as a green kitten. In another, a single fluorescent whisker was the only evidence of infection found. All grades of severity between these two conditions were seen. The face and ears of cats were the most common areas where infection with ringworm could be demonstrated, but this may be due to the fact that these areas are more easily examined than other parts of the body. $\mathrm{La}$ Touche $(1952 b)$ has drawn attention to the fact that ringworm infection in cats may be inapparent in normal light, and only revealed under the Wood lamp. This 
was abundantly confirmed during the present investigation. Many of the cats examined looked perfectly normal, but under the Wood lamp, scattered infected hairs and small fluorescent lesions were easily seen. Several of the animals had palely fluorescent hairs buried in normal areas and only visible when the fur was separated. It seems clear that many more kittens are infected than those in which bald patches appear. La Touche (1955a) has described infection of the claws of cats. No infected claws have as yet been seen in this clinic, but they were not looked for during the earlier years of the survey. Three of the five dogs regarded as definite sources of human infections were available for examination in the clinic. In these animals, the infection was more obvious and could easily be seen in ordinary light. In one puppy annular lesions with concentric rings of scaling were seen. This puppy was seen again some weeks later, and in spite of treatment its infection had spread extensively so that large fluorescent lesions were scattered over the head and ventral surface. In two infected Siamese cats that were brought for examination, the lesions were also much more obvious and resembled those found in the dogs.

\section{ENVIRONMENTAL FACTORS}

\section{Infections among human contacts}

Siblings of patients existed in 114 of the 138 families studied and in $71(62.3 \%)$ of the families, siblings had infections of tinea capitis or tinea corporis. Most of these infections appeared to develop at about the same time, and in almost all the families one or two of the children escaped infection. This bears out the view expressed by Thomas (1953) and others that $M$. canis has a low level of infectivity from child to child. The simultaneous infections suggest a common animal source for the family, while the escape of some siblings shows that even in domestic conditions the infection is not easily contracted. In 14 families, a parent or grandparent was involved, but no adult scalp infections were seen. It was possible to examine large numbers of class contacts in three suburban schools. In none of these were infected contacts found.

\section{SOCIAL STATUS AND HOME CONDITIONS}

Eastcott (personal communication) has classified New Zealand citizens into four social groups, namely: group 0, farm workers of all kinds; group I, professional and managerial; group II, skilled labourers; and group III, unskilled labourers. The work of the breadwinner was recorded in $\mathbf{9 5}$ of the families studied. The distribution of these families within the social groups is shown in Table 2. From the table it will be seen that the breadwinner in $72 \cdot 6 \%$ of the families had a profession or was a skilled labourer. The homes of 75 families were visited, and the home conditions were grouped as good, fair and poor. The conditions were regarded as good in $45(58 \%)$ families, as fair in $25(33 \%)$ and as poor in only $6(9 \%)$ families. The geographical distribution of infected families was also studied, and the infections appeared to be more or less evenly distributed throughout the residential areas of the town. 
Although Carlier (1954) in her eight years' survey of the ringworm flora of Birmingham describes a steady increase of infections due to $M$. canis, she states in her conclusions: 'The stigma attaching to ringworm in the popular mind is not without foundation... . Ringworm is obviously a disease favoured by overcrowding and neglect, both of children and of domestic animals. It is favoured, too, by stupidity and ignorance.' Whatever may be true of infections with $M$. audouini, the facts given above do not support this statement for $M$. canis infections. New Zealand has a high standard of living, and the families in which infections occurred were not those of the lowest income groups. The infections were not confined to particular residential areas, and in the majority of homes visited the conditions were reasonably good. Even in the 6 homes classed as poor, each child had a separate bed, and the grouping as 'poor' was made on the general untidiness of the household, rather than on the population density. La Touche (1952a) has stated 'if infected cats with no visible signs of their microsporosis roam the streets, any child is liable to contamination or possible infection'. This statement seems highly applicable to the situation in Dunedin, and it appears that in this area there should be no stigma attached to an infection caused by $M$. canis.

\section{DISCUSSION AND CONCLUSIONS}

It is not very easy to discuss the results of this investigation because they are not susceptible to rigid analysis. The study was undertaken in the hope that the different clinical patterns of infection could be related to the source of infection. Since $M$. canis is known to lose its communicability after relatively few human passages, it was postulated that an infection acquired from a human source might have a shorter clinical course, and that such a strain of $M$. canis might have less powers of invasion in the individual scalp as well as less communicability to another human host. The results obtained have not supported this hypothesis, and the clinical course of infection does not appear to bear any relation to the source of infection. Nor does the sex or age of the patient appear to affect the course of infection, except in very wide limits. There is evidence to suggest that girls become less susceptible to ringworm more rapidly than do boys, but if a girl does become infected, she seems to be equally likely to have a generalized infection as does her brother. Children of more than 10 years of age seem to have more localized lesions and a shorter clinical course, but below an age approaching puberty, no effect of increasing years could be observed.

There are other possible explanations of the varying clinical patterns. One is that strains of $M$. canis vary in their invasive powers. So far no evidence in support of this theory has been obtained. It seems reasonable to regard the same strain as infecting all the members of a family where simultaneous infections occur, yet within a family one child will have only a local lesion, while a sibling will show a generalized infection.

Another reason for the variation might be the difference in efficiency with which the mother applies the topical application, but once again the different patterns seen within the same family appear to be evidence against this explanation. 
The most reasonable explanation which suggests itself is that there are intrinsic differences in the human scalp which determine the clinical course of the infection. These differences might depend on skin structure, allergic reactions and other factors. Further investigations on these lines are contemplated. The other conclusion which can be drawn from this study is that human ringworms, due to $M$. canis, are part of a complicated network of infection, involving the passage of the parasite among domestic pets themselves as well as among human subjects. The cat appears to be the major reservoir of the infection in New Zealand, and it is probably due to its peculiar relationship to man that the infection is perpetuated. The domestic cat tends to live as a commensal with man. Among cat-loving families it is treated with proper care and attention, but the majority of people seem to regard the cat as an appendage required to provide at least part of its food by catching mice. This attitude of only partial responsibility for the welfare of the animal results in the birth of litters of kittens in hidden corners, distant from the home to which the mother theoretically belongs. These kittens become strays and are adopted by children, and if they are carrying $M$. canis, a human infection may result. It has been frequently suggested that ringworm in cats flourishes in poorly nourished animals, and these semi-wild cat families may well be short of food and therefore particularly susceptible to infection by $M$. canis. The conclusions of Carlier (1954) already quoted may well apply to the cats of this area. It seems probable that greater care by cat owners of their pets, so that kittens are either disposed of, or looked after properly, would be a major factor in the prevention of human ringworm infections.

\section{SUMMARY}

1. The clinical findings and environmental factors of 138 families infected with Microsporum canis are recorded.

2. Of the 171 children suffering from tinea capitis, $25 \%$ had lesions which remained localized, $51 \%$ had local spread of infection and $24 \%$ had a generalized spread, involving most of the scalp.

3. The clinical pattern of the infection was not associated with the sex or age of the child, nor with the source of infection.

4. In $30.5 \%$ of the families there was a definite cat source of infection, in $3.6 \%$ a definite dog source and in $13.9 \%$ a definite human source. A further $\mathbf{2 0 . 3} \%$ had a probable animal source, and $\mathbf{7 . 9} \%$ a probable human source, while in $23.9 \%$ no obvious source of infection could be found.

5. The social status of 95 families was investigated. In $4 \cdot 2 \%$ the breadwinner was a farmer, in $12.6 \%$ he belonged to the professional and managerial group, in $60 \%$ he was a skilled labourer and in $22.1 \%$ an unskilled labourer. The houses of 75 families were visited. House conditions were classed as good in $58 \%$, fair in $33 \%$ and poor in $9 \%$. It is suggested that ringworm due to $M$. canis in New Zealand is not associated with poverty or poor conditions and no stigma should be attached to the development of an infection.

6. Evidence for the following types of transmission of infection was obtained: cat to child; dog to child; child to child; child to adult; cat to cat; child to cat; dog to cat; cat to dog. 
7. The findings of the investigation are discussed. It is suggested that human infections with $M$. canis are part of a complicated network resulting from the commensal relationship of the cat to man. Better care of cats so that kittens are not allowed to stray would assist in the control of human infections.

I would like to acknowledge with gratitude the assistance given me by Miss F. L. Drummond who undertook the home visits. My thanks are also due to the Medical Research Council of New Zealand for financial assistance, to Dr M. E. di Menna and Miss M. E. Bailey for technical help and to Prof. J. A. R. Miles for much advice and encouragement.

\section{REFERENCES}

Carlier, Gwendoline, I. M. (1954). J. Hyg., Camb., 52, 264.

La Touche, C. J. (1952a). Vet. Rec. 64, 398.

La Touche, C. J. (1952b). Brit. med. J. $2,1081$.

LA Touche, C. J. $(1955 a)$. Vet. Rec. 67, 578.

LA Touche, C. J. (1955b). Vet. Rec. 67, 666.

MaRPLeS, M. J. (1951). N.Z. med. J. 50, 460.

Schwartz, L., Peck, S. M., Botviniok, I., Liebowitz, A. L. \& Frasier, Elizabeth (1946). J. Amer. med. Ass. 132, 58.

Thомas, B. A. (1953). Brit. med. J. i, 536.

WALker, JACQUELINE (1950). Brit. J. Derm. Syph. 62, 239.

(M.S. received for publication 24. I. 56) 